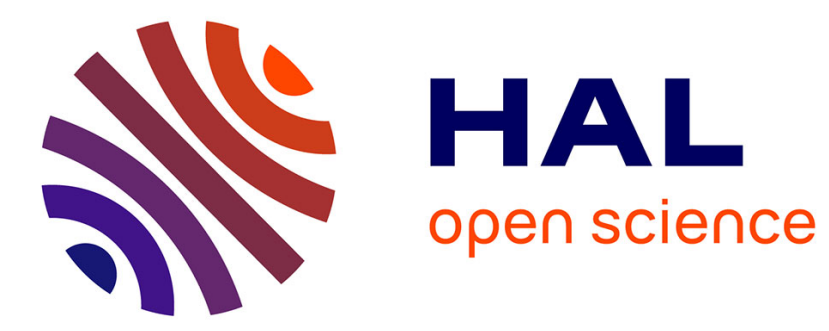

\title{
Inductor shape optimization for electromagnetic casting
}

Alfredo Canelas, Jean Rodolphe Roche, José Herskovits

\section{To cite this version:}

Alfredo Canelas, Jean Rodolphe Roche, José Herskovits. Inductor shape optimization for electromagnetic casting. [Research Report] RR-6733, INRIA. 2008, pp.28. inria-00342050

\section{HAL Id: inria-00342050 \\ https://hal.inria.fr/inria-00342050}

Submitted on 26 Nov 2008

HAL is a multi-disciplinary open access archive for the deposit and dissemination of scientific research documents, whether they are published or not. The documents may come from teaching and research institutions in France or abroad, or from public or private research centers.
L'archive ouverte pluridisciplinaire HAL, est destinée au dépôt et à la diffusion de documents scientifiques de niveau recherche, publiés ou non, émanant des établissements d'enseignement et de recherche français ou étrangers, des laboratoires publics ou privés. 


\title{
I N R I A
}

INSTITUT NATIONAL DE RECHERCHE EN INFORMATIQUE ET EN AUTOMATIQUE

\section{Inductor shape optimization for electromagnetic casting}

\author{
Alfredo Canelas — Jean R. Roche — José Herskovits
}

$N^{\circ} 6733$

Novembre 2008

Thème NUM

apport

de recherche 



\title{
Inductor shape optimization for electromagnetic casting
}

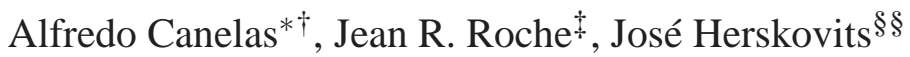 \\ Thème NUM — Systèmes numériques \\ Équipes-Projets CALVI \\ Rapport de recherche $n^{\circ} 6733$ - Novembre 2008 - 28 pages
}

\begin{abstract}
The design of inductors in electromagnetic shaping of molten metals consists of looking for the position and the shape of a set of electric wires such that the induced electromagnetic field makes a given mass of liquid metal acquire a predefined shape. In this paper we formulate an inverse optimization problem where the position and shape of the inductors are defined by a set of design variables. The solution of this problem correspond to the optimal design, i.e., the shape of the liquid metal is as close as possible to the target shape. In a first formulation of the inverse optimization problem we minimize the difference between the target and the equilibrium shapes while in a second approach we minimizes the $L^{2}$ norm of a fictitious surface pressure that makes the target shape to be in mechanical equilibrium. Geometric constraints that prevent the inductors to penetrate into the liquid metal are considered in both formulations. The optimization problems are solved using FAIPA, a line search interior-point algorithm for nonlinear optimization. Some examples are presented to show the effectiveness of the proposed approaches. .
\end{abstract}

Key-words: shape optimization, inverse problems, electromagnetic casting

* Mechanical Engineering Program - COPPE - Federal University of Rio de Janeiro, PO Box 68503, CEP 21945-970, CT, Cidade Universitária, Ilha do Fundão, Rio de Janeiro, Brazil.

† Shared foot note

† I.E.C.N., Nancy-Université, CNRS, INRIA B.P. 239, 54506 Vandoeuvre lès Nancy, France.

$\S$ Mechanical Engineering Program - COPPE - Federal University of Rio de Janeiro, PO Box 68503, CEP 21945-970, CT, Cidade Universitária, Ilha do Fundão, Rio de Janeiro, Brazil.

Centre de recherche INRIA Nancy - Grand Est

LORIA, Technopôle de Nancy-Brabois, Campus scientifique,

615, rue du Jardin Botanique, BP 101, 54602 Villers-Lès-Nancy

Téléphone : +33 383593000 - Télécopie : +33383278319 


\section{Optimisation de la forme des inducteurs en magnétoformage}

Résumé : La conception d'inducteurs dans le formage électromagnétique de métaux liquides consiste en chercher la position et la forme d'un jeu de fils électriques tel que le champ électromagnétique induit fait qu'une masse donnée de métal liquide acquière une forme prédéterminée. Dans ce papier nous formulons un problème d'optimisation inverse où la position et la forme des inducteurs sont définies par un jeu de variables de conception. La solution de ce problème correspond à la conception optimale, c'est-àdire, la forme du métal liquide est aussi près que possible d'une forme prédéterminée. Dans une première formulation du problème d'optimisation inverse nous réduisons au minimum la différence entre l'objectif et les formes en équilibre tandis que dans une deuxième approche nous réduisons au minimum la norme $L^{2}$ d'une pression superficielle factice qui fait en sorte que la forme objectif vérifie l'équilibre mécanique. On considère des contraintes géométriques qui empêchent les inducteurs de pénétrer dans le métal liquide dans les deux formulations. Les problèmes d'optimisation sont résolus utilisant FAIPA, un algorithme de points intérieurs pour l'optimisation non-linéaire. Quelques exemples sont présentés pour montrer l'efficacité des approches proposées.

Mots-clés : problèmes inverses, optimisation de formes, magnétoformage 


\section{Contents}

1 Introduction 3

2 The mathematical model of the electromagnetic shaping problem 5

2.1 The variational model of the direct problem . . . . . . . . . . 6

2.2 First order optimality conditions . . . . . . . . . . . . . 6

\begin{tabular}{|lll}
\hline & The inverse problem & 7
\end{tabular}

3.1 Two approaches for the inverse problem . . . . . . . . . . . . . 9

4 Numerical Method 10

4.1 The exterior Dirichlet problem . . . . . . . . . . . . . . . . 10

4.2 The SAND formulation of the inverse problems . . . . . . . . . . . 11

4.3 The numerical model . . . . . . . . . . . . . . . . . 12

4.3.1 Discretization of the domain . . . . . . . . . . . . . . 12

4.3 .2 Inductors . . . . . . . . . . . . . . . . . . . 12

4.3.3 Exterior boundary value problem . . . . . . . . . . . . . 13

4.3 .4 Equilibrium equation . . . . . . . . . . . . . . . . . . . . . . 14

4.3 .5 Geometric constraints . . . . . . . . . . . . . 15

4.4 Discretized inverse problems . . . . . . . . . . . . . . 16

$\begin{array}{lll}5 & \text { Numerical examples } & 17\end{array}$

5.1 Example $1 \ldots \ldots \ldots \ldots \ldots 17 \ldots \ldots \ldots$

5.2 Example 2 . . . . . . . . . . . . . . . . . . . . . . . . . . . . . . . . . . . . .

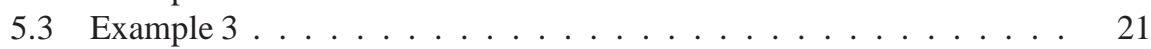

5.4 Example $4 \ldots \ldots \ldots 21$

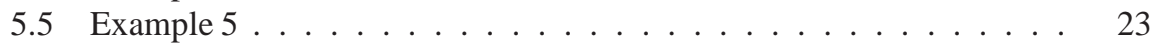

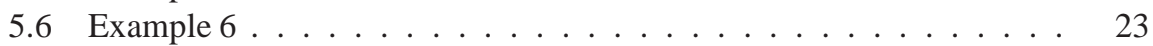

5.7 Summary of results . . . . . . . . . . . . . . . . . 24

6 Conclusions 25

$\begin{array}{lll}7 & \text { Acknowledgements } & 25\end{array}$

\section{Introduction}

Electromagnetic Casting (EMC) and Magnetic Suspension Melt Processing (MSMP) are important technologies in the metallurgical industry. They are based on the repulsive forces that an alternating electromagnetic field produces on the surface of some kind of materials. They make use of the electromagnetic field for contactless heating, shaping and control of solidification of hot melts. The EMC has primarily been employed for containerless continuous casting but is mainly used to prepare ingots of aluminum alloy [15]. Another important application, extensively used in aeronautics, astronautics, energy and chemical engineering, is in the manufacturing of components of engines made of superalloy materials $(\mathrm{Ni}, \mathrm{Ti}, . .$.$) [14]. Advantages of these tech-$ niques are to produce components with high surface quality, high cleanness and low contamination.

The EMC problem studied here concerns the case of a vertical column of liquid metal falling down into an electromagnetic field created by vertical inductors. In Fig. 1 


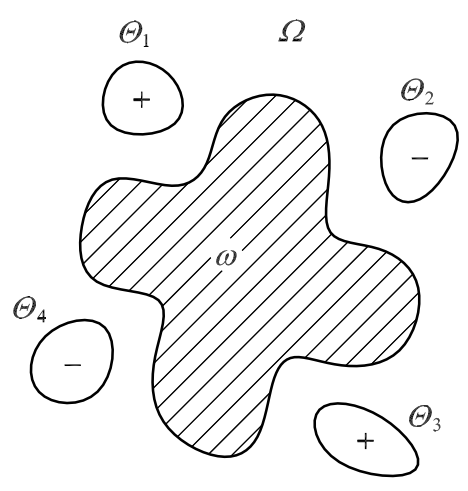

Figure 1: EMC problem.

the horizontal cross-section of the inductors is represented by the domains $\Theta_{p}, 1 \leq$ $p \leq 4$, and the cross-section of the liquid metal is represented in the figure by the domain $\omega$. Given the position and shape of the inductors, the magnetic field created by them produces a surface pressure on the vertical column of liquid metal. That surface pressure forces the liquid metal to change its shape until an equilibrium relation on the boundary between the electromagnetic pressures and surface tensions is satisfied. The boundary shape of the liquid metal such that the equilibrium is attained can be found as the solution of a nonlinear free-surface problem, see [24], [25] for details. Our purpose is to design suitable inductors such that the equilibrium shape of the liquid metal be as close as possible to a given target shape.

In a previous work we studied this EMC problem considering the case where the inductors are made of single solid-core wires with a negligible area of the cross-section [13]. Thus, the inductors were represented by points in the horizontal plane. In this paper we consider the more realistic case where each inductor is a set of bundled insulated strands. In this case we represent the inductors by a domain in the plane as depicted by Fig. 11 The electric current density is assumed uniform on the entire cross-section of the inductor. This is a very reasonable approximation for the case where the inductors are made up of multiple individually insulated strands twisted or woven together.

Our goal is to determine the position and shape of the domains $\Theta_{p}$ that represent the cross-section of the inductors in order to have an horizontal cross-section of the molten metal as close as possible to the prescribed shape. For this purpose we consider the two different approaches proposed in [13]. The first one looks for a set of inductors such that minimizes the distance between the computed shape and the given target one. The second approach minimizes the error of the equilibrium equation for the target shape.

In addition, here we introduce a new technique to consider geometric constrains that prevent the inductors to penetrate the liquid metal. These constraints are more suitable that the box constraints considered in [13] making the considered formulations more effective and robust for the solution of the EMC problem.

In this paper we employ a SAND formulation for both approaches and solve the optimization problems employing the Feasible Arc Interior Point Algorithm, FAIPA, a line search interior-point algorithm for nonlinear optimization. See [32], [33], [42], [37], [6] for a general discussion of the SAND formulation, [5], [34], [12], [13] for some other issues and applications and [20], [34], [21] for details about FAIPA. 


\section{The mathematical model of the electromagnetic shap- ing problem}

We assume that the frequency of the imposed current is very high so that the magnetic field does not penetrate into the metal. In other words we neglect the skin effect. Moreover, we assume that a stationary horizontal section is reached so that the 2-dimensional model is valid. The equilibrium of the system is insured by the static balance on the surface of the metal between the surface tension forces and the electromagnetic forces. This problem and other similar ones have been considered by several authors, we refer the reader to the following papers for the physical analysis of the simplifying assumptions that the above model requires: see [9], [18], [19], [22], [40], [24] 10].

We denote by $\Omega$ the exterior in the plane of the compact and simply connected domain $\omega$ occupied by the cross-section of the metal column; see Fig. 1

The exterior magnetic field can be found as the solution of the following boundary value problem:

$$
\begin{aligned}
\nabla \times \vec{B} & =\mu_{0} \vec{J} & & \text { in } \Omega, \\
\nabla \cdot \vec{B} & =0 & & \text { in } \Omega, \\
\vec{B} \cdot v & =0 & & \text { on } \Gamma, \\
\|\vec{B}\| & =O\left(\|x\|^{-1}\right) & & \text { as }\|x\| \rightarrow \infty \text { in } \Omega .
\end{aligned}
$$

Here the fields $\vec{J}=(0,0, J)$ and $\vec{B}=\left(B_{1}, B_{2}, 0\right)$ represent the mean square values of the current density vector and the total magnetic field, respectively. The constant $\mu_{0}$ is the vacuum permeability, $v$ the unit normal vector to the boundary $\Gamma$ and $\|\cdot\|$ denotes the Euclidean norm. We assume that $J$ has compact support in $\Omega$ and satisfies:

$$
\int_{\Omega} J d x=0
$$

On the other hand, the magnetic field produces a surface pressure that acts on the liquid metal, changing the shape until the equilibrium is attained. This equilibrium is characterized by the following equation [24], [25]:

$$
\frac{1}{2 \mu_{0}}\|\vec{B}\|^{2}+\sigma \mathscr{C}=p_{0} \quad \text { on } \Gamma,
$$

where $\mathscr{C}$ is the curvature of $\Gamma$ seen from the metal, $\sigma$ is the surface tension of the liquid and the constant $p_{0}$ is an unknown of the problem. Physically, $p_{0}$ represents the difference between the internal and external pressures. Since it is assumed that the molten metal is incompressible, we have the following condition:

$$
\int_{\omega} d x=S_{0}
$$

where $S_{0}$ is given.

In the direct problem the electric current density $J$ is given and one needs to find the shape of $\omega$ that satisfies (7) and such that the magnetic field $\vec{B}_{\omega}$ solution of (1)-(4) satisfies also the equilibrium equation (6) for a real constant $p_{0}$.

Conditions 11-5, with the function $J$ compactly supported in $\Omega$, imply that there exists the flux function $\varphi: \Omega \rightarrow \mathbb{R}$ such that $\vec{B}=\left(\frac{\partial \varphi}{\partial x_{2}},-\frac{\partial \varphi}{\partial x_{1}}, 0\right)$ and $\varphi$ is the solution of:

$$
\begin{aligned}
-\Delta \varphi & =\mu_{0} J & & \text { in } \Omega, \\
\varphi & =0 & & \text { on } \Gamma, \\
\varphi(x) & =O(1) & & \text { as }\|x\| \rightarrow \infty .
\end{aligned}
$$




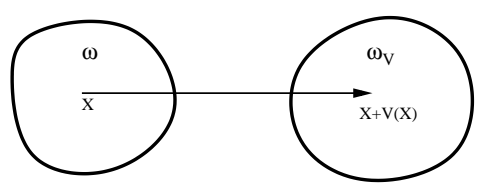

Figure 2: Domain perturbation.

The equilibrium equation (6) in terms of the flux $\varphi$ becomes:

$$
\frac{1}{2 \mu_{0}}\|\nabla \varphi\|^{2}+\sigma \mathscr{C}=p_{0} \quad \text { on } \Gamma .
$$

The direct problem, in terms of the flux, consists of looking for a domain $\omega$ such that the solution $\varphi_{\omega}$ of (8) satisfies (9) for a real constant $p_{0}$.

\subsection{The variational model of the direct problem}

Under suitable assumptions, the equilibrium configurations are given by the local stationary points with respect to the domain of the following total energy:

$$
E(\omega)=-\frac{1}{2 \mu_{0}} \int_{\Omega}\left\|\nabla \varphi_{\omega}\right\|^{2} d x+\sigma P(\omega),
$$

subject to the equality constraint in the measure of $\omega$ :

$$
\int_{\omega} d x=S_{0}
$$

In [10], $\varphi_{\omega}$ is the solution of 8 and $P(\omega)$ is the perimeter of $\omega$, i.e., the length of $\Gamma=\partial \omega$ when $\partial \omega$ is regular enough (for instance of class $C^{1}$ ):

$$
P(\omega)=\int_{\Gamma} d \gamma, \quad d \gamma=\text { length measure on } \Gamma .
$$

The variational formulation of the direct problem consists of finding the domain $\omega$ as a stationary point of the total energy [10, subject to the constraint 111. As $\varphi_{\omega}$ is solution of $[8$, to prove that this variational formulation is equivalent to the previous one it remains to show that the equilibrium relation is automatically ensured for all the stationary points.

\subsection{First order optimality conditions}

In order to derive the first order optimality conditions we consider shape derivatives. Differentiation with respect to the domain is a classical issue, in this work we consider the point of view of F. Murat and J. Simon; see [1], [39], [31].

Let $V \in W^{1, \infty}\left(\mathbb{R}^{2}, \mathbb{R}^{2}\right)$ the set of the Lipschitz functions $\phi$ from $\mathbb{R}^{2}$ to $\mathbb{R}^{2}$ such that $\phi$ and $\nabla \phi$ are uniformly bounded [1]. Let $\omega$ be a bounded domain in $\mathbb{R}^{2}$ of class $C^{2}$. We consider a shape deformation given by the mapping $I d+V$, where $I d$ is the identity mapping. Then, the deformed set $\omega_{V}$ is defined by $\omega_{V}=\{x+V(x) \mid x \in \omega\}$; see Fig. [2

For every $V \in W^{1, \infty}\left(\mathbb{R}^{2}, \mathbb{R}^{2}\right)$ the mapping $I d+V$ is a diffeomorphism provided $\|V\|_{W^{1, \infty}\left(\mathbb{R}^{2}, \mathbb{R}^{2}\right)}<1[1]$. 
Let $\mathscr{O}(\omega)$ be the collection of images of $\omega$ considering all possible diffeomorphisms. If $F$ is a scalar function defined in $\mathscr{O}(\omega)$ we said that it is shape differentiable if the function $V \rightarrow F\left(\omega_{V}\right)$ is differentiable at $V=0$ in the Banach space $W^{1, \infty}\left(\mathbb{R}^{2}, \mathbb{R}^{2}\right)$.

The derivative of $F$, defined in $W^{1, \infty}\left(\mathbb{R}^{2}, \mathbb{R}^{2}\right)$, is called shape gradient and is denoted by $F^{\prime}(\omega)$. It can be shown that the linear application $V \rightarrow F^{\prime}(\omega)(V)$ is determined by the normal component of $V$ in the boundary of $\omega$, see the works by [1], [7] and [40] for a detailed description of the shape derivative structure.

Let $L$ be the Lagrangian function defined in $\mathscr{O}(\omega) \times \mathbb{R}$ by:

$$
L\left(\omega, p_{0}\right)=E(\omega)-p_{0}\left(m(\omega)-S_{0}\right),
$$

Then, the first order optimality condition is the following:

$$
L^{\prime}\left(\omega, p_{0}\right)(V)=0 \quad \forall V \in W^{1, \infty}\left(\mathbb{R}^{2}, \mathbb{R}^{2}\right) .
$$

This kind of optimality conditions often appear in hydrodynamic problems and other fluid problems; let us refer for instance to the work by [10] where a large class of liquid metal equilibria is considered.

Theorem Let $\Omega$ be the complement of a compact set $\omega$ in $\mathbb{R}^{2}$ with nonempty interior. Assume that $\Gamma=\partial \omega=\partial \Omega$ is of class $C^{2}$. Let $V$ be in $W^{1, \infty}\left(\mathbb{R}^{2}, \mathbb{R}^{2}\right)$ with compact support and $\|V\|_{W^{1, \infty}\left(\mathbb{R}^{2}, \mathbb{R}^{2}\right)}<1$. Let $F$ be a square integrable function from $\Omega$ into $\mathbb{R}$ with compact support in $\Omega$.

Then, there exists a unique solution $\varphi_{\omega_{V}}$ in $C^{1}\left(\bar{\Omega}_{V}\right)$ (see [2] and [19]) of:

$$
\begin{aligned}
-\Delta \varphi_{\omega_{v}} & =\mu_{0} J & & \text { in } \Omega_{V}, \\
\varphi_{\omega_{V}} & =0 & & \text { on } \partial \Omega_{V}, \\
\varphi_{\omega_{V}}(x) & =O(1) & & \text { as }\|x\| \rightarrow \infty .
\end{aligned}
$$

and the shape derivative of the lagrangian $L$ is given by:

$$
L^{\prime}\left(\omega, p_{0}\right)(V)=\int_{\Gamma}\left(\frac{1}{2 \mu_{0}}\left\|\nabla \varphi_{\omega}\right\|^{2}+\sigma \mathscr{C}-p_{0}\right)(V \cdot v) d \gamma
$$

where $v$ is the unit normal to $\Gamma$ oriented toward $\Omega, \mathscr{C}$ is the curvature of $\Gamma$ (seen from the metal) and $\varphi_{\omega}$ the solution of (8).

Proof See [19], [24], [40], [1].

This problem is very similar to some ones considered by several authors. We refer the readers to the following papers and references therein for the physical analysis of the simplifying assumptions that the above model requires: see [9], [16], [18], [19], [4], [22], [38], [40], [23], [24], [25], [3], [28], [30], [35], [36].

\section{The inverse problem}

The goal of the inverse problem is to find a distribution of current around the liquid metal column so that it attains a given shape.

Given the target shape $\omega^{*}$, we want to compute $J$ as the solution of the following optimization problem:

$$
\min _{J} \mathrm{~d}\left(\omega, \omega^{*}\right),
$$

where the function $d$ is a distance between $\omega$ and $\omega^{*}$. The domain $\omega$ belongs to the set of admissible domains, i.e., $\omega \in \mathscr{O}$, and is in equilibrium under the action of the 
electric current density $J$ in the variational sense. In other words, $\omega$ satisfies the area constraint 111 and the flux $\varphi_{\omega}$ solution of $[8$ satisfies the equilibrium equation 18 for a real constant $p_{0}$ :

$$
\begin{aligned}
& \int_{\Gamma}\left(\frac{1}{2 \mu_{0}}\left\|\nabla \varphi_{\omega}\right\|^{2}+\sigma \mathscr{C}-p_{0}\right)(V \cdot v) d \gamma=0 \\
& \forall V \text { in } C^{1}\left(\mathbb{R}^{2}, \mathbb{R}^{2}\right) .
\end{aligned}
$$

From a practical point of view, the magnetic field has to be created by a simple configuration of inductors. For that purpose, we consider a distribution of the electric current density $J$ of the form:

$$
J=I \sum_{p=1}^{m} \alpha_{p} \chi_{\Theta_{p}}
$$

where $I$ is a given intensity of current, $\Theta_{p}$, with $1 \leq p \leq m$, are subsets of $\mathbb{R}^{2}, \chi_{\Theta_{p}}$ are their characteristic functions, and $\alpha_{p}$ are dimensionless coefficients. Then, the inverse problem consists of determining the sets $\Theta_{p}$.

Note that the expression 19 assumes that the electric current density is uniform on each region $\Theta_{p}$. Inductors made of bundled insulated strands allow the use of 19] as a good approximation, see [8] and references therein. They are also suitable to make inductors of specific geometries.

For an electric current density given by (19), (5) is satisfied imposing:

$$
\sum_{p=1}^{m} \alpha_{p} \int_{\Theta_{p}} d x=0
$$

Remark 1. In certain cases it is possible to find a current density distribution such that the target shape $\omega^{*}$ is in equilibrium. This topic was already studied and there are a few papers about the existence of such solutions. See [19], [17], [26].

In the two-dimensional case, $\omega^{*}$ is assumed simply connected and its boundary is only one Jordan curve $\Gamma$. [19], show that a solution of [1], [2], 3], 4], and [6, with $J$ compactly supported in $\Omega$ can be found for each $p_{0}$ satisfying:

$$
p_{0} \geq \sigma \max _{x \in \Gamma} \mathscr{C}(x)
$$

That is, assuming $J$ compactly supported in $\Omega$, and choosing $p_{0}$ satisfying 21 , then there exists $\vec{B}$ satisfying (1), (2), (3), (4), and (6) if an only if:

(i) $\Gamma$ is an analytic curve.

(ii) If $p_{0}$ is chosen satisfying the equality in 21], the global maximum of the curvature must be attained in an even number of points.

Moreover, the magnetic field is well determined in a neighborhood of $\omega$ (local uniqueness).

Equation (5) is also obtained if $p_{0}$ is chosen satisfying the equality in (21). A current density distribution concentrated on a curve in $\Omega$ can always be found. However, a solution given by the addition of a finite number of characteristic functions like (19) may be not possible. See [19]. 


\subsection{Two approaches for the inverse problem}

We propose two different approaches for finding an approximate solution of problem 17. The first one considers a domain deformation of $\omega^{*}$ defined by the mapping:

$$
T_{Z}(x)=x+Z(x), \quad \forall x \in \mathbb{R}^{2},
$$

where $Z$ is a regular vector field with compact support in $\mathbb{R}^{2}$. Then, defining:

$$
\begin{aligned}
\omega_{Z} & =T_{Z}\left(\omega^{*}\right) \\
\Gamma_{Z} & =T_{Z}\left(\Gamma^{*}\right)
\end{aligned}
$$

The first inverse formulation is:

$$
\begin{aligned}
& \min _{J, Z}\|Z\|_{L^{2}\left(\Gamma^{*}\right)}^{2}, \\
& \text { subject to: } \\
& \omega_{Z} \text { is in equilibrium under } J .
\end{aligned}
$$

A second formulation of the inverse problem can be considered introducing a slack variable function $p(x): \Gamma \rightarrow \mathbb{R}$ in order to make the equilibrium equation satisfied for the target shape:

$$
\begin{array}{r}
\int_{\Gamma^{*}}\left(\frac{1}{2 \mu_{0}}\left\|\nabla \varphi_{\omega}\right\|^{2}+\sigma \mathscr{C}-p_{0}+p\right)(V \cdot v) d \gamma=0 \\
\forall V \text { in } C^{1}\left(\mathbb{R}^{2}, \mathbb{R}^{2}\right) .
\end{array}
$$

The function $p$ can be understood as an additional pressure acting on the interface. Given $J$ and $\omega^{*}, p$ is the surface pressure that equilibrates the action of the magnetic pressure and the surface tension. The second formulation for the inverse problem is an indirect approach that try to minimize the $L^{2}\left(\Gamma^{*}\right)$ norm of the function p:

$$
\begin{aligned}
& \min _{J, p}\|p\|_{L^{2}\left(\Gamma^{*}\right)}^{2}, \\
& \text { subject to: } \\
& \omega^{*} \text { is in equilibrium under } J \text { and } p .
\end{aligned}
$$

Remark 2. In this last formulation only shape variables concerning the inductors are considered. This fact makes 25 much easier to solve than 23. If the function $p$ vanishes at the solution of 25, the resulting electric current density $J$ will also be a solution of the first formulation with the equilibrium domain matching exactly the target shape. In the general case, $p$ will not vanish at the solution and, in this case, the target shape $\omega^{*}$ will not be in equilibrium under $J$ only. Then, a second stage of analysis will be necessary to find the equilibrium domain under the obtained current density distribution. However, as the norm of $p$ was minimized, the resultant equilibrium domain is expected to be a good approximation of the target one. Furthermore, since 25. can be solved with a minor computational effort, its solution $J$ can be employed as an initial guess for the formulation 23 . 


\section{Numerical Method}

\subsection{The exterior Dirichlet problem}

To solve (8) in the exterior domain $\Omega$ we consider a particular solution $\varphi_{1}$ of the differential equation given by:

$$
\varphi_{1}(x)=-\frac{\mu_{0}}{2 \pi} \int_{\mathbb{R}^{2}} \ln \|x-y\| J(y) d y .
$$

This function is a solution of the problem:

$$
\begin{aligned}
-\Delta \varphi_{1}(x) & =\mu_{0} J \quad \text { in } \mathbb{R}^{2}, \\
\varphi_{1}(x) & =O(1) \quad \text { as }\|x\| \rightarrow \infty .
\end{aligned}
$$

Note that for the current density distribution defined by [19, the expression of $\varphi_{1}$ is

$$
\varphi_{1}(x)=-\frac{\mu_{0} I}{2 \pi} \sum_{p=1}^{m} \alpha_{p} \int_{\Theta_{p}} \ln \|x-y\| d y .
$$

The function $\varphi_{1}$ can be calculated as a sum of line integrals on the boundaries $\Gamma_{p}$ of domains $\Theta_{p}$. Consider the function $w: \mathbb{R}^{2} \times \mathbb{R}^{2} \rightarrow \mathbb{R}^{2}$ defined as:

$$
w(x, y)=(1 / 4)(1-2 \ln \|x-y\|)(x-y) .
$$

The divergence of $w$ is $\nabla_{y} \cdot w=\ln \|x-y\|$. Then, 29 becomes:

$$
\varphi_{1}(x)=-\frac{\mu_{0} I}{2 \pi} \sum_{p=1}^{m} \alpha_{p} \int_{\Gamma_{p}} w(x, y) \cdot v d \gamma
$$

The function $\varphi$ can be computed as:

$$
\varphi(x)=\xi(x)+\varphi_{1}(x),
$$

where the function $\xi$ is the solution of the following exterior problem:

$$
\begin{aligned}
-\Delta \xi(x) & =0 & & \text { in } \Omega, \\
\xi(x) & =-\varphi_{1}(x) & & \text { on } \Gamma, \\
\|\xi(x)\| & =O(1) & & \text { as }\|x\| \rightarrow \infty .
\end{aligned}
$$

Following [2], an integral single layer representation of the solution of (33) is given by:

$$
\xi(x)=-\frac{1}{2 \pi} \int_{\Gamma} q(y) \ln \|x-y\| d \gamma+c,
$$

where the constant $c$ is the value at the infinity of $\xi$ and the function $q(y) \in H^{-1 / 2}(\Gamma)$ satisfies:

$$
\int_{\Gamma} q(y) d \gamma=0
$$

It remains to impose the boundary conditions on $\Gamma$. Here, this is done with a weak formulation. Let $a_{\Gamma}(q, g)$ be the following elliptic bilinear form:

$$
a_{\Gamma}(q, g)=-\frac{1}{2 \pi} \int_{\Gamma} g(x) \int_{\Gamma} q(y) \ln \|x-y\| d \gamma d \gamma+
$$




$$
+c \int_{\Gamma} g(x) d \gamma
$$

defined on $H^{-1 / 2}(\Gamma) \times H^{-1 / 2}(\Gamma)$. We look for a function $q(y) \in H^{-1 / 2}(\Gamma)$ that satisfies (35) and:

$$
a_{\Gamma}(q, g)=-\int_{\Gamma} \varphi_{1}(x) g(x) d \gamma \quad \forall g \in H^{-1 / 2}(\Gamma) .
$$

Finally, the norm $\|\nabla \varphi\|$ in (18) can be computed as:

$$
\|\nabla \varphi\|=\left|\frac{\partial \varphi}{\partial v}\right|=\left|\frac{\partial \varphi_{1}}{\partial v}+\frac{\partial \xi}{\partial v}\right|,
$$

where the first equality comes from the fact that $\varphi$ is constant on $\Gamma$, and the second one from 32. The normal derivative of $\varphi_{1}$ is obtained from 31:

$$
\frac{\partial \varphi_{1}}{\partial v_{x}}(x)=-\frac{\mu_{0} I}{2 \pi} \sum_{p=1}^{m} \alpha_{p} \int_{\Gamma_{p}} \frac{\partial}{\partial v_{x}}(w(x, y) \cdot v) d \gamma .
$$

The following expression can be used for $\xi$ :

$$
\frac{\partial \xi}{\partial v_{x}}(x)=-\frac{1}{2 \pi} \int_{\Gamma} q(y) \frac{\partial}{\partial v_{x}} \ln \|x-y\| d \gamma+\frac{1}{2} q(x) \quad \forall x \in \Gamma,
$$

where the integral of 40 is understood in the Cauchy principal value sense.

\subsection{The SAND formulation of the inverse problems}

A SAND formulation of the inverse problems 23 and 25 is employed here. In other words, the state variables $p_{0}, c$ and $q$ are incorporated as unknowns of the optimization problem and the state and equilibrium equations are incorporated as equality constraints. The optimization problem of the formulation 23 becomes:

$$
\min _{J, Z, p_{0}, c, q}\|Z\|_{L^{2}\left(\Gamma^{*}\right)}^{2}
$$

subject to the area constraint:

$$
\int_{\omega_{Z}} d x=S_{0}
$$

the state equations:

$$
\begin{gathered}
a_{\Gamma_{Z}}(q, g)=-\int_{\Gamma_{Z}} \varphi_{1}(x) g(x) d \gamma \quad \forall g \in H^{-1 / 2}\left(\Gamma_{Z}\right), \\
\int_{\Gamma_{Z}} q(y) d \gamma=0,
\end{gathered}
$$

and the equilibrium equation:

$$
\begin{aligned}
& \int_{\Gamma_{Z}}\left(\frac{1}{2 \mu_{0}}\|\nabla \varphi\|^{2}+\sigma \mathscr{C}-p_{0}\right)(V \cdot v) d \gamma=0 \\
& \forall V \text { in } C^{1}\left(\mathbb{R}^{2}, \mathbb{R}^{2}\right),
\end{aligned}
$$

where $\varphi_{1}, \varphi$, and $\xi$ are given by (26, 32) and 34 . 
The optimization problem of the formulation 25 becomes:

$$
\min _{J, p, p_{0}, c, q}\|p\|_{L^{2}\left(\Gamma^{*}\right)}^{2}
$$

subject to the state equations:

$$
\begin{gathered}
a_{\Gamma^{*}}(q, g)=-\int_{\Gamma^{*}} \varphi_{1}(x) g(x) d \gamma \quad \forall g \in H^{-1 / 2}\left(\Gamma^{*}\right), \\
\int_{\Gamma^{*}} q(y) d \gamma=0,
\end{gathered}
$$

and the equilibrium equation:

$$
\begin{aligned}
& \int_{\Gamma^{*}}\left(\frac{1}{2 \mu_{0}}\|\nabla \varphi\|^{2}+\sigma \mathscr{C}-p_{0}+p\right)(V \cdot v) d \gamma=0 \\
& \forall V \text { in } C^{1}\left(\mathbb{R}^{2}, \mathbb{R}^{2}\right) .
\end{aligned}
$$

\subsection{The numerical model}

\subsubsection{Discretization of the domain}

We consider an approximation of the domain $\omega^{*}$ defined by the piecewise linear closed boundary $\Gamma^{h}$, i.e., $\Gamma^{h}$ is the union of the $n$ linear finite elements $\ell_{j}$ in $\mathbb{R}^{2}, j \in\{1, \ldots, n\}$. The nodes of the boundary $\Gamma^{h}$ are denoted by $x_{i}$.

A direction $\hat{Z}^{i} \in \mathbb{R}^{2}$ is associated to each vertex $x_{i}$ of $\Gamma^{h}$. We construct a continuous piecewise linear vector field $Z^{i}$ from $\Gamma^{h}$ in $\mathbb{R}^{2}$ such that $Z^{i}\left(x_{k}\right)=\delta_{i k} \hat{Z}^{i}$. The support of $Z^{i}$ is equal to the union of the finite elements for which $x_{i}$ is a node. The vector field $Z$ of (22) is computed as:

$$
Z(x)=\sum_{i=1}^{n} u_{i} Z^{i}(x),
$$

and the updated boundary $\Gamma_{\vec{u}}$ is then given by:

$$
\Gamma_{\vec{u}}=\left\{X \mid X=x+Z(x) ; u_{i} \in \mathbb{R}, x \in \Gamma^{h}\right\},
$$

where $\vec{u}^{T}=\left(u_{1}, \ldots, u_{n}\right) \in \mathbb{R}^{n}$ is the vector of unknowns which determine the evolution of the boundary. This representation has the advantage of defining only one degree of freedom for each node. We denote by $\omega_{\vec{u}}$ the interior domain related to $\Gamma_{\vec{u}}$ in order to show the dependence with respect to the vector $\vec{u}$.

\subsubsection{Inductors}

Each inductor has a geometry corresponding to one of the parametric shapes given by Fig. 3 The contribution of each inductor to the function $\varphi_{1}$ is calculated using 31. The boundary $\Gamma_{p}$ of each inductor is divided in small linear elements to perform the integration. The entire set of shape parameters corresponding to the inductors is denoted by $\vec{u}_{p}$. 


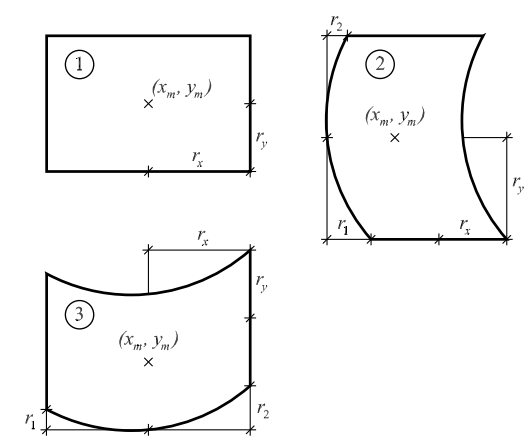

Figure 3: Geometry of the inductors and shape parameters. 1. four-parameter inductor of rectangular shape; 2. six-parameter inductor of parabolic vertical sides; 3. sixparameter inductor of parabolic horizontal sides.

\subsubsection{Exterior boundary value problem}

For numerical calculations we consider a piecewise constant approximation $q_{h}(x)$ of $q(x)$ :

$$
q_{h}(x)=\sum_{j=1}^{n} q_{j} e_{j}(x)
$$

where $e_{j}(x)=1$ if $x \in \ell_{j}$ and zero elsewhere.

Replacing the function $g$ in 43 by $e_{i}$, with $i \in\{1, \ldots, n\}$, the weak formulation of the boundary value problem, given by equations (43) and 44), becomes:

$$
\vec{A}(\vec{u}) \vec{q}=\vec{b}\left(\vec{u}_{p}, \vec{u}\right),
$$

where the vector $\vec{q}^{T}=\left(q_{1}, \ldots, q_{n}, c\right)$ is in $\mathbb{R}^{n+1}, \vec{u}$ is the vector of shape variables and $\vec{u}_{p}$ is the vector that contains the shape parameters of the inductors. The coefficients $a_{i j}$ of the symmetric matrix $\vec{A}(\vec{u})$ are:

$$
\begin{aligned}
& a_{i j}(\vec{u})=-\frac{1}{2 \pi} \int_{\ell_{i}} \int_{\ell_{j}} \ln \|x-y\| d \gamma d \gamma \quad i, j \in\{1, \ldots, n\} \\
& a_{i j}(\vec{u})=\int_{\ell_{j}} d \gamma \quad i=n+1, \text { and } j \in\{1, \ldots, n\}
\end{aligned}
$$

and the components $b_{i}$ of the vector $\vec{b}$ are:

$$
\begin{array}{rlrl}
b_{i}\left(\vec{u}_{p}, \vec{u}\right) & =-\int_{\ell_{i}} \varphi_{1}(x) d \gamma & & i \in\{1, \ldots, n\}, \\
b_{i}\left(\vec{u}_{p}, \vec{u}\right)=0 & i=n+1,
\end{array}
$$

Remark 3. For given vectors $\vec{u}$ and $\vec{u}_{p}$, the linear system (53) is symmetric and nonsparse. Numerical approximations of the element integrals of previous and later equations are computed by Gauss quadrature. 
Remark 4. If $q$ is the solution of the system 35, 37) and the piecewise constant approximation $q_{h}$ given by the solution of [53, then we have the following error bounds (see [41]):

$$
\left\|q-q_{h}\right\|_{H^{-1 / 2}(\Gamma)} \leq C_{1} h\|q\|_{H^{1}(\Gamma)},
$$

and if $\xi_{h}$ is the approximation of 34 then

$$
\left\|\frac{\partial \xi}{\partial v}-\frac{\partial \xi_{h}}{\partial v}\right\|_{H^{-1 / 2}(\Gamma)} \leq C_{2} h\|q\|_{H^{1}(\Gamma)} .
$$

Remark 5. The approximation of the normal derivative $\frac{\partial \xi}{\partial v}$ at $x_{l} \in \ell_{l}$ is given by:

$$
\frac{\partial \xi_{h}}{\partial v}\left(x_{l}\right)=-\frac{1}{2 \pi} \sum_{\substack{i=1 \\ i \neq l}}^{n} q_{i} \sum_{m=1}^{K} p_{m} \frac{\partial \ln \left\|x_{l}-x_{i}\left(s_{m}\right)\right\|}{\partial v}+\frac{1}{2} q_{l}
$$

where $x_{i}\left(s_{m}\right)$ are the integration points and $p_{m}$ the weights of the Gauss quadrature formula. Thus, the computation of $\frac{\partial \xi_{h}}{\partial v}\left(x_{l}\right)$ needs $O(n)$ floating point operations.

\subsubsection{Equilibrium equation}

Consider a direction $\hat{V}^{i} \in \mathbb{R}^{2}$ associated to each vertex $x_{i}$ of $\Gamma^{h}$ and the continuous piecewise linear vector field $V^{i}$ from $\Gamma^{h}$ in $\mathbb{R}^{2}$ such that $V^{i}\left(x_{k}\right)=\delta_{i k} \hat{V}^{i}$. If we project the equation (45) in the finite dimensional space generated by $V^{i}, i \in\{1, \ldots, n\}$, the discrete version of the equilibrium is the following:

$$
\begin{array}{r}
D E_{i}\left(\vec{u}_{p}, \vec{u}, \vec{q}, p_{0}\right)=\int_{\Gamma_{\vec{u}}}\left(\frac{1}{2 \mu_{0}}\|\nabla \varphi\|^{2}-p_{0}\right)\left(V^{i} \cdot v\right) d \gamma+ \\
+\sigma \mathscr{C}^{i} \cdot \hat{V}^{i}
\end{array}
$$

where $i \in\{1, \ldots, n\}$ and $\mathscr{C}^{i}$ is an approximation of the mean curvature at $x_{i}$, given by:

$$
\mathscr{C}^{i}=\left(\frac{\left(x_{i}-x_{i-1}\right)}{\left\|x_{i}-x_{i-1}\right\|}-\frac{\left(x_{i+1}-x_{i}\right)}{\left\|x_{i+1}-x_{i}\right\|}\right) .
$$

The gradient $\nabla \varphi$ is computed using [38, 39] and (40).

In the case of equation (49), we consider a piecewise linear function $p_{h}$ defined as:

$$
p_{h}(x)=\sum_{i=1}^{n} p_{i} f_{i}
$$

where the function $f_{i}$ satisfies $f_{i}\left(x_{k}\right)=\delta_{i k}$. Then, defining $\vec{p}^{T}=\left(p_{1}, \ldots, p_{n}\right)$, the equilibrium equation is defined as:

$$
\begin{gathered}
D F_{i}\left(\vec{u}_{p}, \vec{p}, \vec{q}, p_{0}\right)=\frac{1}{2 \mu_{0}} \int_{\Gamma^{*}}\left(\|\nabla \varphi\|^{2}-p_{0}+p_{h}\right)\left(V^{i} \cdot v\right) d \gamma+ \\
+\sigma \mathscr{C}^{i} \cdot \hat{V}^{i}
\end{gathered}
$$




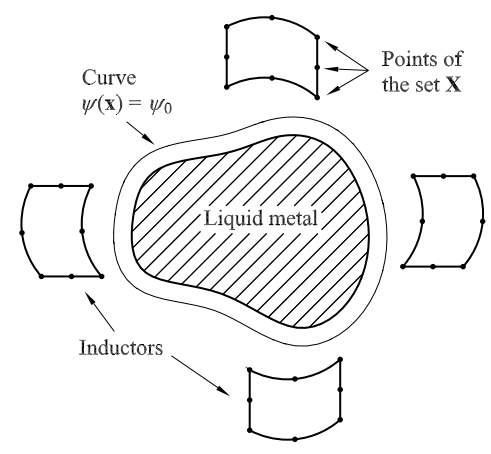

Figure 4: Geometric constraints.

\subsubsection{Geometric constraints}

Some geometric constraints are needed to prevent the penetration of the inductors into the liquid metal. That is done here considering the following inequalities:

$$
\psi\left(x_{j}\right) \leq \psi_{0}, \quad \text { for all } x_{j} \in \vec{X}
$$

where $\vec{X}$ is a chosen set of points belonging to the boundary of the inductors. The real valued function $\psi$ is zero in the interior of the liquid metal and negative in the exterior. Then, choosing a negative value for the parameter $\psi_{0}, 65$ enforces the points $x_{j}$ to be in the exterior of the liquid metal as illustrated by Fig. 4

The function $\psi$ is defined as the solution of:

$$
\begin{array}{rlrl}
\Delta \psi(x) & =0 & & \text { in } \Omega^{*}, \\
\psi(x) & =0 & & \text { on } \Gamma^{*}, \\
\int_{\Gamma^{*}} \nabla \psi(x) \cdot v d \gamma & =-1 . &
\end{array}
$$

Like function $\xi$ of Section $4.1 \psi$ can be calculated as:

$$
\psi(x)=-\frac{1}{2 \pi} \int_{\Gamma} q(y) \ln \|x-y\| d \gamma+c
$$

where $q$ must satisfy:

$$
\int_{\Gamma} q(y) d \gamma=-1
$$

In a similar way as in Section 4.3.3 an approximated solution of $q$ and $c$ can be obtained solving a linear system similar to 53 . The numerical approximation of the function $\psi$ is obtained employing 67.

The value $\psi_{0}$ can be defined choosing a point in the exterior of the liquid metal and calculating the value of the function $\psi$ at this point. See Figs. 5 and 6 that show the function $\psi$ for two different target shapes.

Defining $\vec{h}_{j}\left(\vec{u}_{p}\right)=\psi\left(x_{j}\left(\vec{u}_{p}\right)\right)-\psi_{0}$, all the geometric constraints are expressed as:

$$
\vec{h}\left(\vec{u}_{p}\right) \leq 0 .
$$




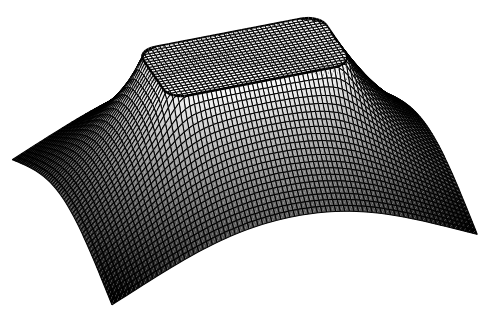

Figure 5: Function $\psi$ for a rectangular like target shape.

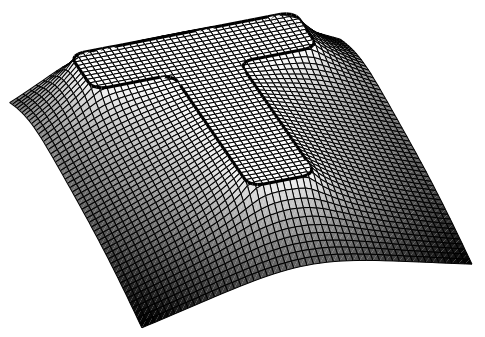

Figure 6: Function $\psi$ for a "T" like target shape.

\subsection{Discretized inverse problems}

Let the area function be $S(\vec{u})=\int_{\omega_{\vec{u}}} d x$, and $D E\left(\vec{u}_{p}, \vec{u}, \vec{q}, p_{0}\right)$ the vector function such that $(D E)_{i}=D E_{i}\left(\vec{u}_{p}, \vec{u}, \vec{q}, p_{0}\right)$. The discretized version of the first inverse problem is the following:

$$
\min _{\vec{u}_{p}, \vec{u}, \vec{q}, p_{0}}\|Z\|_{L^{2}\left(\Gamma^{*}\right)}^{2}
$$

subject to the nonlinear equality constraints:

$$
\left(\begin{array}{c}
\vec{A}(\vec{u}) \vec{q}-\vec{b}\left(\vec{u}_{p}, \vec{u}\right) \\
S(\vec{u})-S_{0} \\
D E\left(\vec{u}_{p}, \vec{u}, \vec{q}, p_{0}\right)
\end{array}\right)=0,
$$

and the nonlinear inequalities:

$$
\vec{h}\left(\vec{u}_{p}\right) \leq 0
$$

The discretized version of the second inverse problem is:

$$
\min _{\vec{u}_{p}, \vec{q}, c, p_{0}}\|p\|_{L^{2}\left(\Gamma^{*}\right)}^{2}
$$

with the equality constraints:

$$
\left(\begin{array}{c}
\vec{A} \vec{q}-\vec{b}\left(\vec{u}_{p}\right) \\
D F\left(\vec{u}_{p}, \vec{p}, \vec{q}, p_{0}\right)
\end{array}\right)=0
$$

and the nonlinear inequalities:

$$
\vec{h}\left(\vec{u}_{p}\right) \leq 0 .
$$

In this case, since the integrals are defined on the fixed domain $\Gamma^{*}$ the vector $\vec{u}$ of shape variables is not present in the formulation. 


\section{Numerical examples}

We consider several examples to illustrate the behavior of the proposed formulations of the inverse problem. The goal is to identify the position and shape of the inductors given by the shape variables $\vec{u}_{p}$. The shape and the surface $S_{0}$ of the target shape, the surface tension $\sigma$, the intensity $I$ and the dimensionless coefficients $\alpha_{p}$ are given. For each example all the parameters, including the parameters $\psi_{0}$ of the geometric constraints, are the same for both formulations. The initial values of the state variables $\vec{q}$ and $p_{0}$, the shape variable $\vec{u}$ of the first formulation and the pressure $\vec{p}$ of the second one are set equal to zero for all the examples.

For the solution of the optimization problems, the line search interior-point algorithm for nonlinear constrained optimization problems FAIPA was employed. For a given feasible point with respect to the inequality constrains, FAIPA defines a feasible and descent arc solving three linear systems of equations with the same coefficient matrix. Then, it performs a line search along this arc to define the next iterate. FAIPA makes subsequent iterations until a certain convergence criterion is satisfied. For more details about FAIPA see [20], [34], [21]. The number of iterations of FAIPA was limited to 400 in all the examples.

For each example we plot the initial position and shape of the inductors, the target shape of the liquid metal and shape of the inductors obtained by the optimization algorithm.

\subsection{Example 1}

The target shape of this example is the solution of the direct free-surface problem considering four concentrated intensities of value $I=0.1$, with the sign given by Fig. [7] see [Example 1a] [13].

For the inverse problem we consider four inductors of type 1 of Fig. 3. and a target shape of area $S_{0}$ equal to $\pi$. The intensity $I$ is equal to 0.1 and the surface tension $\sigma$ is equal to $1.0 \times 10^{-4}$. The dimensionless coefficients $\alpha_{p}$ have absolute value equal to 4.0 with the sign given by Fig. 8 , two configurations for the initial positions of the inductors, named Ex1a and Ex1b, are considered as depicted by the figure.

The configuration of inductors obtained was the same for both initial configurations and both formulations. The equilibrium shape obtained is almost the same that the target one and none of the geometric constraints is active at the solution. Fig. 9 shows the inductors obtained and some level curves of the flux function $\varphi$ at the solution.

Employing the first formulation and starting from the initial configuration of Fig. 8 a, the optimization algorithm found the solution in just 7 iterations, but otherwise the number of iterations was large when starting from the configuration of Fig. $8 \mathrm{~b}$. When the second formulation was used, the algorithm solves the problem at very reasonable cost, and with a very good accuracy as Table 1 shows.

\subsection{Example 2}

In this example the target shape is the rounded square depicted by Fig. 10 For the inverse problem we consider four inductors of type 1 of Fig. 3 and a target shape of area $S_{0}$ equal to 3.86. The intensity $I$ is equal to 0.1 and the surface tension $\sigma$ is equal to $1.0 \times 10^{-4}$. The dimensionless coefficients $\alpha_{p}$ have absolute value equal to 4.0 with the sign given by Fig. 10 Four different values of the parameter $\psi_{0}$ of the geometric constraints are considered, these values generate four different problems that we have 


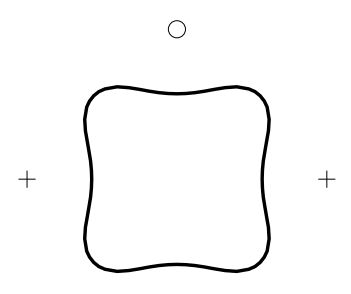

$\bigcirc$

Figure 7: Example 1, target shape considering concentrated intensities. Solid line: equilibrium shape, plus: positive current, circle: negative current.

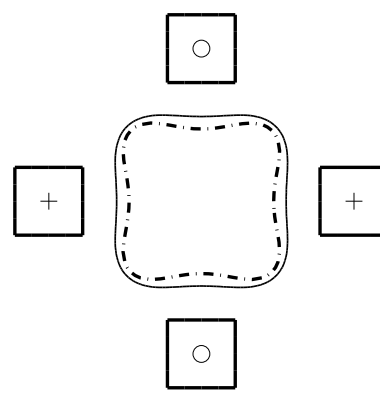

a
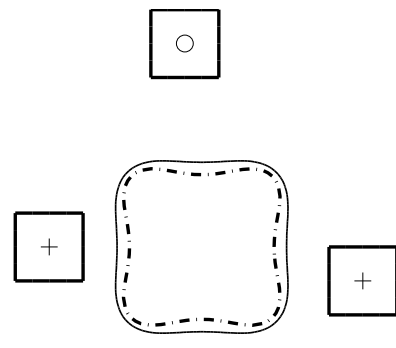

b

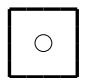

Figure 8: Example 1, initial configuration and geometric constraints of examples Ex1a and Ex1b, a example Ex1a. b example Ex1b. Dash-dot line: target shape, solid line: curve $\psi(x)=\psi_{0}$, plus: inductor of positive current, circle: inductor of negative current. 


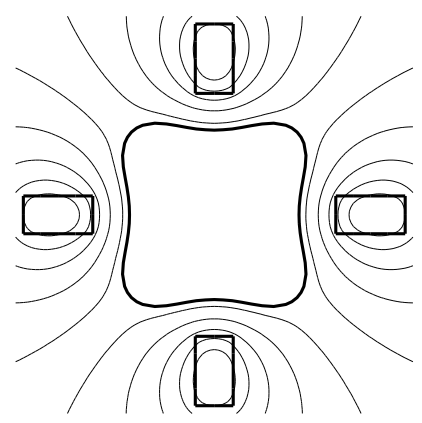

Figure 9: Solution of the Example 1, both formulations, equilibrium shape and level curves of the flux function $\varphi$.

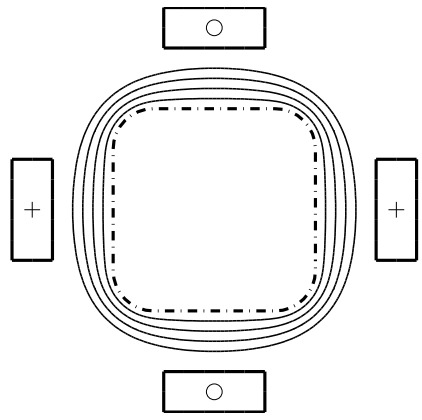

Figure 10: Example 2, initial configuration and geometric constraints of examples Ex2a to Ex2d. Dash-dot line: target shape, solid line: curves $\psi(x)=\psi_{0}$ for four different values of $\psi_{0}$, plus: inductor of positive current, circle: inductor of negative current.

named Ex2a to Ex2d, as shown by the Fig. 10 The example Ex2a corresponds to the constraint given by the closest curve to the target shape while the example Ex $2 \mathrm{~d}$ corresponds to the the farthest one.

For the first formulation, the same configuration of the inductors was obtained for all cases as shown by Fig. 11] a; the equilibrium shape and some level curves of the flux function $\varphi$ at the solution is depicted by Fig. 11 b. The geometric constraints were not active in all the examples using this formulation. Employing the second formulation, the final configuration of inductors depends on the value of the parameter $\psi_{0}$. For the larger value, Fig. 12] a shows the inductors obtained and Fig. 12]b depicts the equilibrium shape and some level curves of the flux function $\varphi$ at the solution. Figure 13 shows the same for the smaller value of $\psi_{0}$. Different from the first formulation, the second one has the solution having the inductors as close as the liquid metal as possible. In the four cases the geometric constraint is active. Although the location of the inductors is quite different using one or the other formulation, the optimum value of the objective function of the first formulation is almost the same for all the results obtained. 


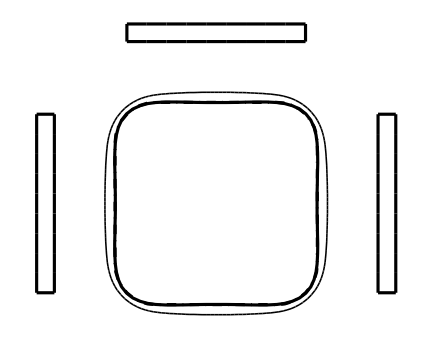

a

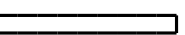

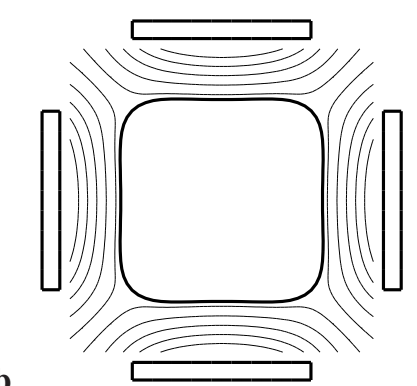

b

Figure 11: Solution of example Ex2a, first formulation, a solution and geometric constraint, $\mathbf{b}$ equilibrium shape and level curves of the flux function $\varphi$.

$\mathbf{a}$

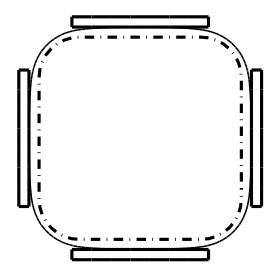

b

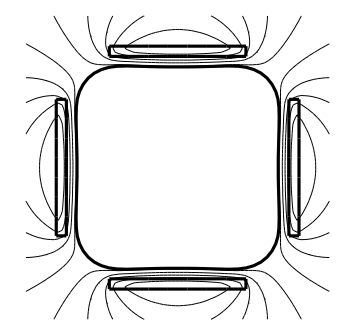

Figure 12: Solution of example Ex2a, second formulation, a solution and geometric constraint, $\mathbf{b}$ equilibrium shape and level curves of the flux function $\varphi$.

$\mathbf{a}$

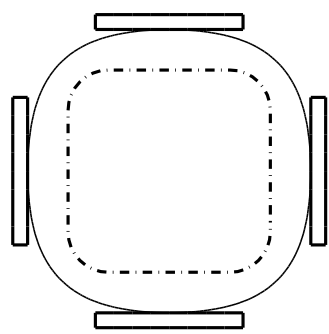

b

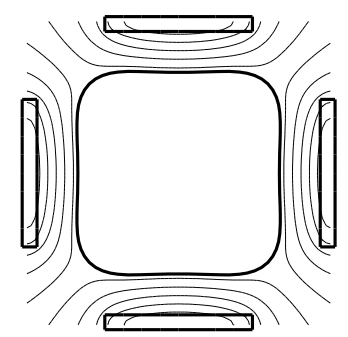

Figure 13: Solution of example Ex2d, second formulation, a solution and geometric constraint, $\mathbf{b}$ equilibrium shape and level curves of the flux function $\varphi$. 


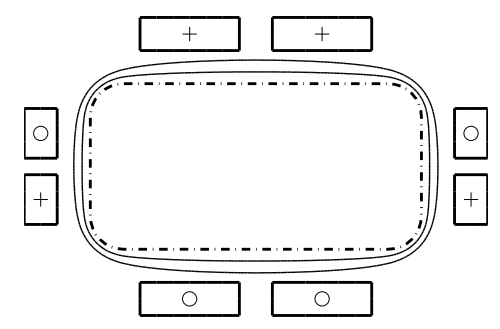

Figure 14: Example 3, initial configuration and geometric constraints of examples Ex3a and Ex3b. Dash-dot line: target shape, solid line: curves $\psi(x)=\psi_{0}$ for four different values of $\psi_{0}$, plus: inductor of positive current, circle: inductor of negative current.

$\mathbf{a}$
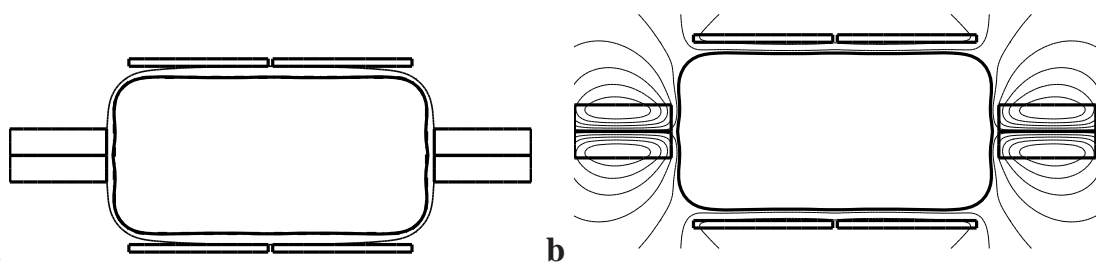

Figure 15: Solution of example Ex3a, first formulation, a solution and geometric constraint, $\mathbf{b}$ equilibrium shape and level curves of the flux function $\varphi$.

\subsection{Example 3}

The target shape of this example is the bar depicted by Fig. 14 For the inverse problem we consider eight inductors of type 1 of Fig. 3. and a target shape of area $S_{0}$ equal to 7.86. The intensity $I$ is equal to 0.1 and the surface tension $\sigma$ is equal to $1.0 \times 10^{-4}$. The dimensionless coefficients $\alpha_{p}$ have absolute value equal to 4.0 with the sign given by Fig. 14 The solutions for two different values of the parameter $\psi_{0}$, named Ex3a and Ex $3 \mathrm{~b}$, are compared.

In this example, the main difference employing one or the other formulation is the size of the inductors located on left and right side. This size depends strongly on the value of the parameter $\psi_{0}$ when using the first formulation but weekly employing the second one. For the larger value and for the first formulation, Fig. 15] a shows the inductors obtained and Fig. 15 b depicts the equilibrium shape and some level curves of the flux function $\varphi$ at the solution. Figures $16 \mathrm{a}$ and $16 \mathrm{~b}$ show the same for the second formulation.

\subsection{Example 4}

This example has the only difference with respect to the previous one in the sign of the coefficients $\alpha_{p}$ as depicted by Fig. 17 For the larger value of the parameter $\psi_{0}$ and for the first formulation, Fig. 18, a shows the inductors obtained and Fig. 18] b depicts the equilibrium shape and some level curves of the flux function $\varphi$ at the solution. Figures 19 a and 19 b show the same for the second formulation. 


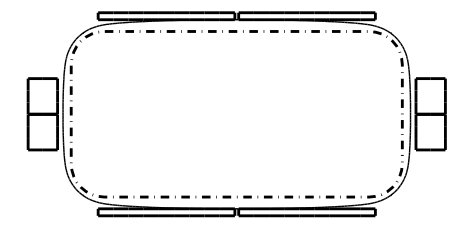

b

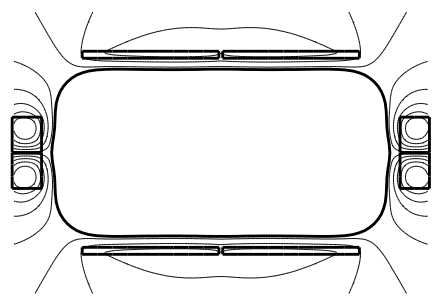

Figure 16: Solution of example Ex3a, second formulation, a solution and geometric constraint, $\mathbf{b}$ equilibrium shape and level curves of the flux function $\varphi$.

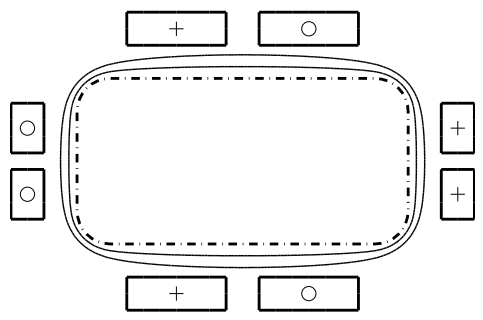

Figure 17: Example 4, initial configuration and geometric constraints of examples Ex4a and Ex4b. Dash-dot line: target shape, solid line: curves $\psi(x)=\psi_{0}$ for four different values of $\psi_{0}$, plus: inductor of positive current, circle: inductor of negative current.

a

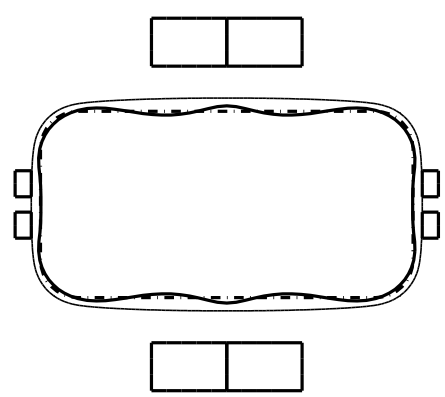

b

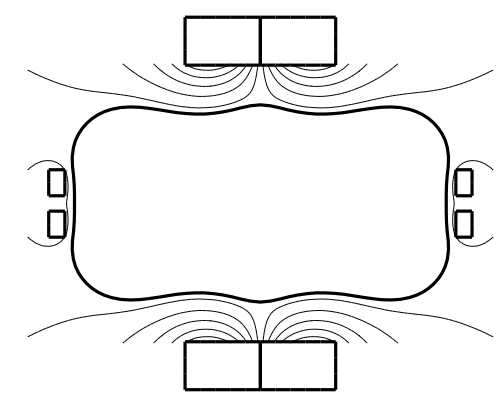

Figure 18: Solution of example Ex4a, first formulation, a solution and geometric constraint, b equilibrium shape and level curves of the flux function $\varphi$.

a

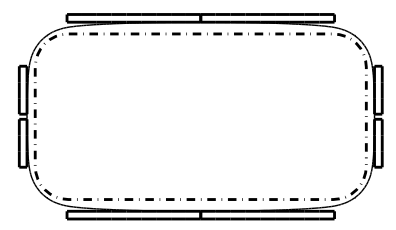

b

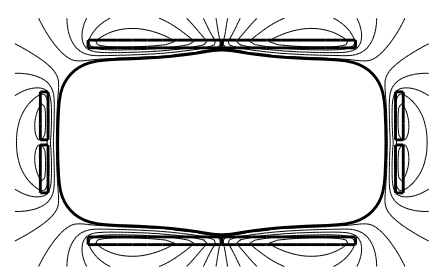

Figure 19: Solution of example Ex4a, second formulation, a solution and geometric constraint, $\mathbf{b}$ equilibrium shape and level curves of the flux function $\varphi$. 


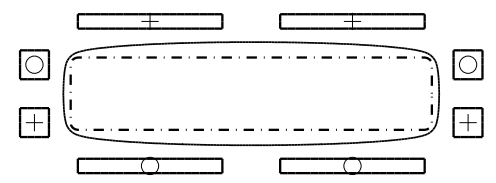

Figure 20: Example 5, initial configuration. Dash-dot line: target shape, solid line: curves $\psi(x)=\psi_{0}$ for four different values of $\psi_{0}$, plus: inductor of positive current, circle: inductor of negative current.
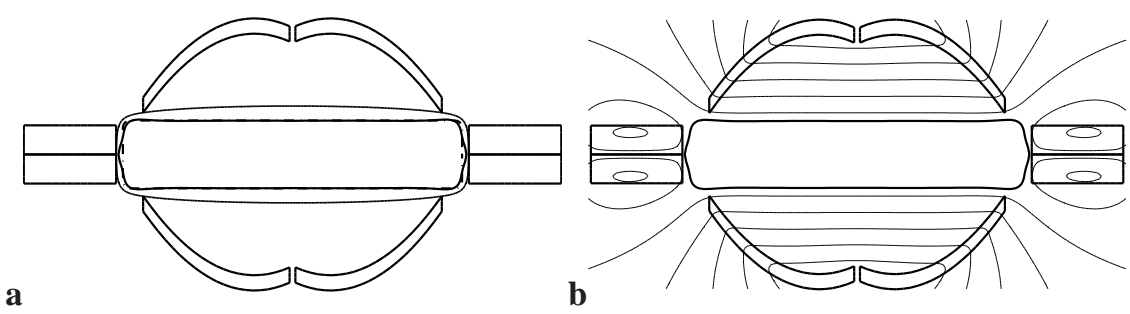

Figure 21: Solution of the example Ex5, first formulation, a solution and geometric constraint, $\mathbf{b}$ equilibrium shape and level curves of the flux function $\varphi$.
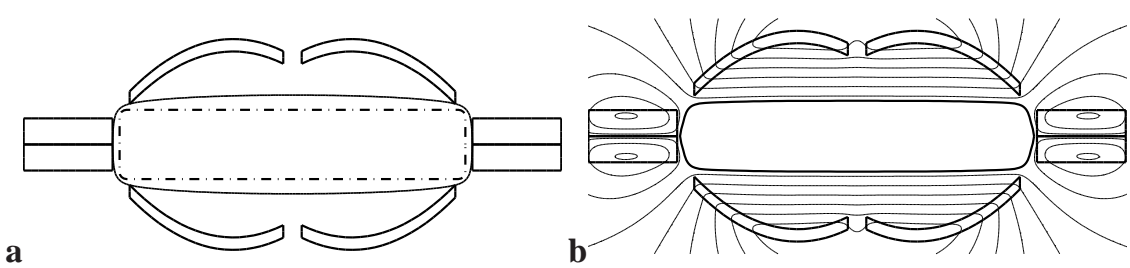

Figure 22: Solution of the example Ex5, second formulation, a solution and geometric constraint, $\mathbf{b}$ equilibrium shape and level curves of the flux function $\varphi$.

\subsection{Example 5}

The target shape of this example is the bar depicted by Fig. 20 For the inverse problem we consider eight inductors. The inductors on the top and bottom side are of type 3 of Fig. 3 while the inductors on the left and right side are of type 1. The target shape has $S_{0}$ equal to 4.99 , the intensity $I$ is equal to 0.1 and the surface tension $\sigma$ is equal to $1.0 \times 10^{-4}$. The dimensionless coefficients $\alpha_{p}$ have absolute value equal to 4.0 with the sign given by Fig. 20

The optimization algorithm was unable to solve this example employing the first formulation. A large distortion of the mesh related to the liquid metal was observed in the last iterations. For the second formulation, Fig. 22] a shows the inductors obtained and Fig. 22 b depicts the equilibrium shape and some level curves of the flux function $\varphi$ at the solution.

\subsection{Example 6}

This example has the only difference with respect to the previous one in the sign of the coefficients $\alpha_{p}$ as depicted by Fig. 23 For the larger value of the parameter $\psi_{0}$ and 


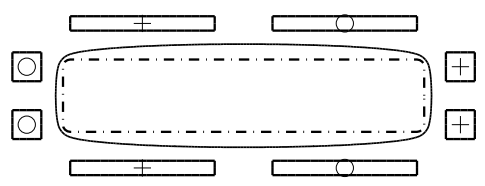

Figure 23: Example 6, initial configuration. Dash-dot line: target shape, solid line: curves $\psi(x)=\psi_{0}$ for four different values of $\psi_{0}$, plus: inductor of positive current, circle: inductor of negative current.

$\mathbf{a}$

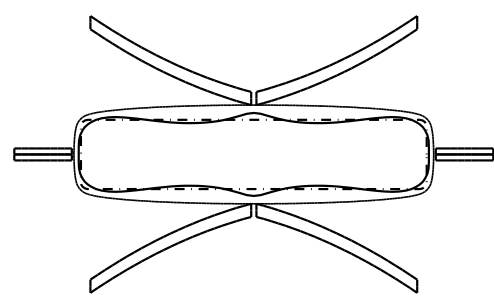

b

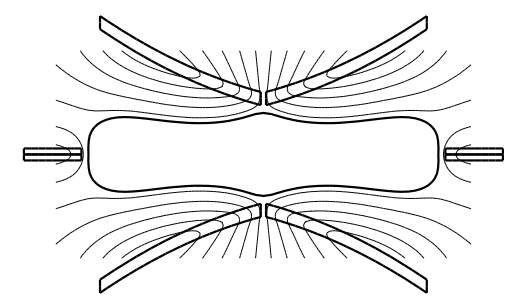

Figure 24: Solution of the example Ex6, first formulation, a solution and geometric constraint, $\mathbf{b}$ equilibrium shape and level curves of the flux function $\varphi$.

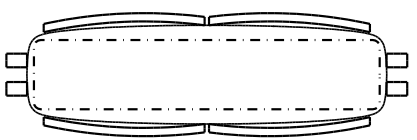

b

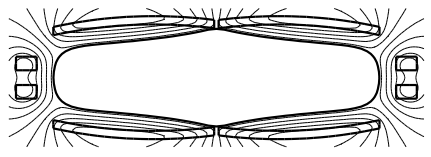

Figure 25: Solution of the example Ex6, second formulation, a solution and geometric constraint, $\mathbf{b}$ equilibrium shape and level curves of the flux function $\varphi$.

for the first formulation, Fig. 24 a shows the inductors obtained and Fig. 24 b depicts the equilibrium shape and some level curves of the flux function $\varphi$ at the solution. Figures 25 a and 25] b show the same for the second formulation. As the figures show, the solutions of the considered formulations are very different in this case. That shows that the results of the second formulation have to be used with caution, its solution can be far different from the best design of the first formulation.

\subsection{Summary of results}

Table 1 resumes the information about the considered examples. For each one we give the number of nodes used for the finite element approximation of the boundary $\Gamma^{*}$ of the target shape and the number of inductors. For each formulation the number of iterations performed by the optimization algorithm is indicated as well as the final value of the objective function. For the second formulation it is also indicated the final value of the objective function of the first formulation, it was calculated solving the freesurface problem considering the inductors obtained by the optimization algorithm. As one could expect, the first formulation shows smaller values of its objective function in all the examples with the only exception of the examples Ex1a and Ex1b where the shape of the inductors at the solution is almost the same. 
Table 1: Summary of results.

\begin{tabular}{lccccccc}
\hline Example & Nodes & Inductors & \multicolumn{2}{c}{ First formulation } & \multicolumn{3}{c}{ Second formulation } \\
& & & Iterations & Obj. F1 ${ }^{\mathrm{b}}$ & Iterations $^{\text {Obj. F1 }}{ }^{\mathrm{b}}$ & Obj. F2 \\
\hline Ex1a & 72 & 4 & 42 & $4.663 \mathrm{e}-07$ & 48 & $1.773 \mathrm{e}-07$ & $3.787 \mathrm{e}-11$ \\
Ex1b & 72 & 4 & 58 & $7.267 \mathrm{e}-07$ & 114 & $1.791 \mathrm{e}-07$ & $3.777 \mathrm{e}-11$ \\
Ex2a & 80 & 4 & 104 & $1.507 \mathrm{e}-04$ & 96 & $1.640 \mathrm{e}-04$ & $2.338 \mathrm{e}-08$ \\
Ex2b & 80 & 4 & 104 & $1.507 \mathrm{e}-04$ & 331 & $1.723 \mathrm{e}-04$ & $3.535 \mathrm{e}-08$ \\
Ex2c & 80 & 4 & 105 & $1.507 \mathrm{e}-04$ & 322 & $1.964 \mathrm{e}-04$ & $4.268 \mathrm{e}-08$ \\
Ex2d & 80 & 4 & 106 & $1.507 \mathrm{e}-04$ & 104 & $2.223 \mathrm{e}-04$ & $4.731 \mathrm{e}-08$ \\
Ex3a & 120 & 8 & 49 & $7.345 \mathrm{e}-04$ & 400 & $1.135 \mathrm{e}-02$ & $1.005 \mathrm{e}-07$ \\
Ex3b & 120 & 8 & 400 & $4.513 \mathrm{e}-03$ & 57 & $1.857 \mathrm{e}-02$ & $1.142 \mathrm{e}-07$ \\
Ex4a & 120 & 8 & 372 & $2.119 \mathrm{e}-02$ & 24 & $9.728 \mathrm{e}-02$ & $1.273 \mathrm{e}-07$ \\
Ex4b & 120 & 8 & 16 & $7.712 \mathrm{e}-02$ & 27 & $2.261 \mathrm{e}-01$ & $1.663 \mathrm{e}-07$ \\
Ex5 & 136 & 8 & 249 & $8.558 \mathrm{e}-03$ & 153 & $2.110 \mathrm{e}-02$ & $8.988 \mathrm{e}-07$ \\
Ex6 & 136 & 8 & 400 & $5.513 \mathrm{e}-02$ & 400 & $5.921 \mathrm{e}-01$ & $1.260 \mathrm{e}-06$ \\
\hline
\end{tabular}

(b) Objective function of the first formulation calculated by a free-surface analysis considering the inductors obtained.

(a) F1: first formulation, F2: second formulation.

\section{Conclusions}

This paper deals with the shape design of inductors concerning the electromagnetic casting of molten metals. Two different approaches based on nonlinear optimization has been proposed in order to find the position and shape of suitable inductors. The first one minimizes the difference between the geometries of the best possible equilibrium domain and the target shape; the second minimizes a slack variable function related to the equilibrium equation on the target boundary. We have also shown how to consider geometric constraints that prevent the inductors to penetrate into the liquid metal. The finite dimensional optimization problems obtained after discretization were solved employing the line search interior-point algorithm FAIPA.

Some presented examples show that both formulations are effective to design suitable inductors. However, the formulations are not equivalent judging by the results obtained for the examples Ex2a-Ex2d and Ex6. The first formulation has shown to be more convenient due to the fact that it found better solutions for almost all the examples. The second formulation could find solutions with similar optimal value of the objective function in most of the cases, but the last presented example has shown that its solution can be, qualitatively, far different from the best design of the first formulation. However, it is less time consuming because of the lack of the shape variables related to the liquid metal. Thus, as most of the results are similar, this formulation appear to be interesting for finding an initial guess for the first formulation.

\section{Acknowledgements}

The authors would like to thank the Brazilian Research Councils CAPES, CNPq and Faperj for the financial support. The first author is supported by the grant E-26/100.280/2007 of Faperj. The second author likes to thank the French Research Councils COFECUB, INRIA and CNRS for the financial support. 


\section{References}

[1] Grégoire, Allaire, Conception Optimale de Structures,Mathématiques and Applications, 58, Springer-Verlag, Berlin, 2007.

[2] Rainer, Kress, Linear integral equations, Applied Mathematical Sciences, 82, Second, Springer-Verlag, New York, 1999.

[3] Jean R. Roche, Algorithmes numériques en optimization de formes et électromagnétisme, , Nancy, Mémoire d'Habilitation à Diriger des Recherches, 1996.

[4] , A. Henrot, J. -P. Brancher and M. Pierre, Existence of equilibria in electromagnetic casting, Proceedings of the Fifth International Symposium on Numerical Methods in Engineering, Vol. 1, 2 (Lausanne, 1989),221-228, Comput. Mech., Southampton, 1989.

[5] Wolfgang Achtziger, On simultaneous optimization of truss geometry and topology, Struct. Multidiscip. Optim.,Structural and Multidisciplinary Optimization,33, 4-5, 285-304, 2007.

[6] S. I. Yi, J.K. Shin and G. J. Park, Comparison of MDO methods with mathematical examples, Struct. Multidiscip. Optim., Structural and Multidisciplinary Optimization, 35, 5, 391-402, 2008.

[7] Arian Novruzi and Michel Pierre, Structure of shape derivatives, Journal of Evolution Equations, 2, 3, 365-382, 2002.

[8] Charles R. Sullivan, Optimal Choice for Number of Strands in a Litz-Wire Transformer Winding, IEEE Transactions On Power Electronics, 14,2, 283-291, 1999.

[9] J.-P. Brancher, J.-P. and O. E. Séro-Guillaume, Etude de la déformation d'un liquide magnétique, Archive for Rational Mechanics and Analysis, 90, 1, 57-85, 1985.

[10] J. A. Shercliff, Magnetic shaping of molten metal columns, Modélisation mathématique et analyse numérique, Proc. R. Soc. Lond., A 375, 455-473, 1981

[11] Olivier Coulaud, Asymptotic analysis of magnetic induction with high frequency for solid conductors, RAIRO Modélisation Mathématique et Analyse Numérique, 32, 6, 651-669, 1998.

[12] A. Canelas, J. Herskovits, J. and J.C.F. Telles, Shape Optimization using the Boundary Element Method and a SAND Interior Point Algorithm for Constrained Optimization, Computers \& Structures, 86, 13-14, 1517-1526, 2007.

[13] Alfredo Canelas, Jean R. Roche and José Herskovits, The inverse electromagnetic shaping problem., Structural and Multidisciplinary Optimization, 2008.

[14] H. Z. Fu, J. Shen, L. Liu, Q.T. Hao, S. M. Li, and J.S. Li, Electromagnetic shaping and solidification control of Ni-base superalloys under vacuum, Journal of Materials Processing Technology, 148, 1, 25-29, 2004.

[15] Zhiqiang, Cao and Fei, Jia and Xingguo, Zhang and Hai, Hao and Junze, Jin, Microstructures and mechanical characteristics of electromagnetic casting and direct-chill casting 2024 aluminum alloys, Materials Science and Engineering A, $327,2,133-137,2002$ 
[16] Olivier Coulaud and Antoine Henrot, Numerical approximation of a free boundary problem arising in electromagnetic shaping, SIAM Journal on Numerical Analysis, 31, 4, 1109-1127, 1994.

[17] Felici, T. P. and Brancher, J.-P., The inverse shaping problem, European Journal of Mechanics. B Fluids, 10, 5, 501-512, 1991.

[18] Gagnoud, A. and Etay, J. and Garnier, M., Le problème de frontière libre en lévitation électromagnétique, Journal de mécanique théorique et appliquée, 5,6,911934, 1986.

[19] Antoine Henrot and Michel Pierre, Un probléme inverse en formage de métaux liquides, Modélisation mathématique et analyse numérique, 23, 1, 155-177, 1989.

[20] José Herskovits, Feasible direction interior-point technique for nonlinear optimization, Journal of Optimization Theory and Applications, 99, 1, 121-146, 1998.

[21] Herskovits, José and Laporte, Emmanuel and Le Tallec, Patrick and Santos, Gines, A quasi-Newton interior point algorithm applied to constrained optimum design in computational fluid dynamics, Revue Européenne des Éléments Finis. European Journal of Finite Elements, 5, 5-6, 595-617, 1996.

[22] H. K. Moffatt, Magnetostatic equilibria and analogous Euler flows of arbitrarily complex topology. I. Fundamentals, Journal of Fluid Mechanics, 159, 359-378, 1985.

[23] Novruzi, A. and Roche, J. R., Newton's method in shape optimisation: a threedimensional case, BIT. Numerical Mathematics, 40,1, 102-120, 2000.

[24] Pierre, M. and Roche, J.-R., Computation of free surfaces in the electromagnetic shaping of liquid metals by optimization algorithms, European Journal of Mechanics. B Fluids, 10, 5, 489-500,1991.

[25] Pierre, Michel and Roche, Jean-R., Numerical simulation of tridimensional electromagnetic shaping of liquid metals, Numerische Mathematik, 65, 2,203$217,1993$.

[26] Pierre, Michel and Rouy, Elisabeth, A tridimensional inverse shaping problem, Communications in Partial Differential Equations, 21, 7-8, 1279-1305, 1996.

[27] Roche, Jean R., Adaptive Newton-like method for shape optimization, Control and Cybernetics, 34, 1, 363-377,2005.

[28] Roche, Jean R., Gradient of the discretized energy method and discretized continuous gradient in electromagnetic shaping simulation, Applied Mathematics and Computer Science, 7, 3, 545-565,1997.

[29] Roche, Jean R. and Sokołowski, Jan, Numerical methods for shape identification problems, Shape optimization and scientific computations (Warsaw, 1995), Control and Cybernetics, 25, 5, 867-894,1996.

[30] Séro-Guillaume, O. E. and Zouaoui, D. and Bernardin, D. and Brancher, J.-P., The shape of a magnetic liquid drop, Journal of Fluid Mechanics, 241, 215-232,1992. 
[31] Simon, J., Differentiation with respect to the domain in boundary value problems, Numerical Functional Analysis and Optimization, 2, 7-8, 649-687, 1980.

[32] Haftka, R. T., Simultaneous analysis and design, AIAA Journal, 23, 7,1099$1103,1985$.

[33] AUTHOR = Haftka, R. T. and Kamat, M. P., Simultaneous nonlinear structural analysis and design, Computational Mechanics, 4, 6, 409-416,1989.

[34] Herskovits, J. and Mappa, P. and Goulart, E. and Mota Soares, C. M., Mathematical programming models and algorithms for engineering design optimization, Computer Methods in Applied Mechanics and Engineering, 194, 30-33, 32443268,2005 .

[35] Sneyd, A. D. and Moffatt, H. K., Fluid dynamical aspects of the levitation-melting process, Journal of Fluid Mechanics, 117, 45-70,1982.

[36] Zouaoui, D. and Séro-Guillaume, O. E. and Brancher, J.-P., Equilibrium of a magnetic liquid drop: variational approach and computation, Magnitnaya Gidrodinamika, 4, 32-35, 150, 1990.

[37] Arora, J. S. and Wang, Q., Review of formulations for structural and mechanical system optimization, Structural and Multidisciplinary Optimization, 30, 4, 251272,2005.

[38] Novruzi, A., Contribution en Optimization de Formes et Applications, Université Henri Poincaré, Nancy 1, 1997.

[39] Murat, S. and Simon, J., Sur le contrôle par un domaine géométrique, Nø76015, Laboratoire d'Analyse Numérique, Université de Paris, 1976.

[40] Novruzi, A. and Roche, J. R., Second Order Derivatives, Newton Method, Application to Shape Optimization, RR-2555, INRIA, 1995

[41] Nédélec, J. C., Approximation des équations intégrales en mécanique et en physique, Centre de mathématiques appliquées -Ecole Polytechnique, 1977.

[42] Arora, J. S. and Wang, Q., Optimization of Large-Scale Structural Systems Using Sparse SAND Formulations, Optimal Design Lab/CCAD, College of Engineering/4110 SC, The University of Iowa, Iowa City, IA 52242, 2004. 
Centre de recherche INRIA Nancy - Grand Est

LORIA, Technopôle de Nancy-Brabois - Campus scientifique

615, rue du Jardin Botanique - BP 101 - 54602 Villers-lès-Nancy Cedex (France)

Centre de recherche INRIA Bordeaux - Sud Ouest : Domaine Universitaire - 351, cours de la Libération - 33405 Talence Cedex Centre de recherche INRIA Grenoble - Rhône-Alpes : 655, avenue de l'Europe - 38334 Montbonnot Saint-Ismier

Centre de recherche INRIA Lille - Nord Europe : Parc Scientifique de la Haute Borne - 40, avenue Halley - 59650 Villeneuve d'Ascq

Centre de recherche INRIA Paris - Rocquencourt : Domaine de Voluceau - Rocquencourt - BP 105 - 78153 Le Chesnay Cedex

Centre de recherche INRIA Rennes - Bretagne Atlantique : IRISA, Campus universitaire de Beaulieu - 35042 Rennes Cedex

Centre de recherche INRIA Saclay - Île-de-France : Parc Orsay Université - ZAC des Vignes : 4, rue Jacques Monod - 91893 Orsay Cedex

Centre de recherche INRIA Sophia Antipolis - Méditerranée : 2004, route des Lucioles - BP 93 - 06902 Sophia Antipolis Cedex 